\title{
MODELLING OF MAGNETORHEOLOGICAL DAMPER FOR INTELLIGENT BIONIC LEG AND SIMULATION OF KNEE JOINT MOVEMENT CONTROL
}

\author{
Xie, H. L.; Liu, Z. B.; Yang, J. Y.; Sheng, Z. Q. \& Xu, Z. W. \\ School of Mechanical Engineering and Automation, Northeastern University, 110004 Shenyang, \\ Liaoning, PR China \\ E-Mail: hlxie@mail.neu.edu.cn, zhiboneu@163.com,jyyang@mail.neu.edu.cn, \\ zhqsheng@me.neu.edu.cn, zhwxu@me.neu.edu.cn
}

\begin{abstract}
To compensate for missing functions of lower extremity amputees, this paper proposes a model of an intelligent bionic leg semi-actively controlled by a magnetorheological damper (MRD). A mechanical structure of the leg is designed, and with the help of Bouc-Wen model, a MRD forward dynamics model is constructed for simulation. On the basis of data obtained from simulations, the MRD inverse dynamics model is constructed by BP neural network. An integrated control platform is constructed for the intelligent bionic leg, on which the simulation for knee joint control is conducted. Results of the research show that the MRD forward dynamics model can precisely express relationships among damping force, velocity, and displacement, while the inverse forward dynamics model can accurately predict MRD control currents, thus obtaining precise trajectory tracking effects of knee joint movement.
\end{abstract}

(Received, processed and accepted by the Chinese Representative Office.)

Key Words: Intelligent Bionic Leg, Magnetorheological Damper, Forward Dynamics Model, Inverse Dynamics Model, RBF Neural Network

\section{INTRODUCTION}

Millions of people living today have lost limbs, especially in the lower extremities, due to a variety of causes such as war, earthquakes, illness, work-related injuries, traffic accidents and accidental injury. Data from the Second National Sample Survey on Disability in 2006 [1] shows that there are 24.12 million Chinese people living with some form of physical disability, accounting for $29.07 \%$ of the total number of the disabled. There are 2.26 million amputees, which is $8 \%$ of physically disabled people. $70 \%$ of amputees are lower extremity amputees. In the United States, there are about 1.6 million people who have lost limbs [2, 3]. Current biomedical technologies cannot regenerate human tissue. Prosthesis $[4,5]$ is the most ideal way to compensate for walking functions of amputated lower extremities. As a highlyintelligent lower-extremity prosthesis, the intelligent bionic leg integrates information technology, electronic technology, control technology, biomedical technology and mechanical technology. It can simulate normal movement of a healthy leg to a great extent, thus helping lower extremity amputees gain mobility and greater independence. Theoretically, this reflects social progress and a heightened awareness and compassion in an effort to relieve pain suffering of amputees. Practically, this can reduce the amount of caregivers needed for the disabled.

As a new intelligent semi-active controller, MRD [6] has already been widely used in shock absorption technology for high speed rail transport and bridges due to its low energy consumption, fast response, and adjustable damping forces with an extensive range. Considering the features of MRD, although some scholars [7-9] have already integrated MRD into knee joint control of intelligent prosthesis, they have not utilized inverse dynamics modelling in this endeavour. Therefore, it fails to gain the intensity required currently for damper effect to achieve desired damping forces. 
The multi-axis knee joint $[10,11]$ of the intelligent bionic leg is nonlinear in movement and is complicated to control. Commonly-used control algorithms include model-based control, PID control, self-adaption control, robust control and iterative control. Model-based control can hardly satisfy the needs of actual application as it requires a mathematical model with real robots. For PID control, ensuring good dynamic and static quality is difficult. Robust control requires the maximum limit value for uncertainty. For iterative control, maintaining stability and convergence is difficult. In contrast, with a simple structure and strong generalization, radial basis function (RBF) network can meet the requirement of realtime control with its high precision of control and its ability to approach any nonlinear function at any degree of precision in a certain compact set.

This paper proposes a model of an intelligent bionic leg semi-actively controlled by MRD and describes the design of its mechanical structure. It also constructs a forward dynamics model for MRD, on the basis of which the inverse dynamics model based on BP neural network is established. An integrated control platform is constructed for the intelligent bionic leg, on which the simulation for knee joint control is employed with a BRP neural network control algorithm. This provides the theoretical foundation for knee joint movement control of the intelligent bionic leg based on MRD.

\section{MECHANICAL STRUCTURE DESIGN FOR INTELLIGENT BIONIC LEG}

The prototype of the intelligent bionic leg developed by the present research group is shown in Fig. 1.

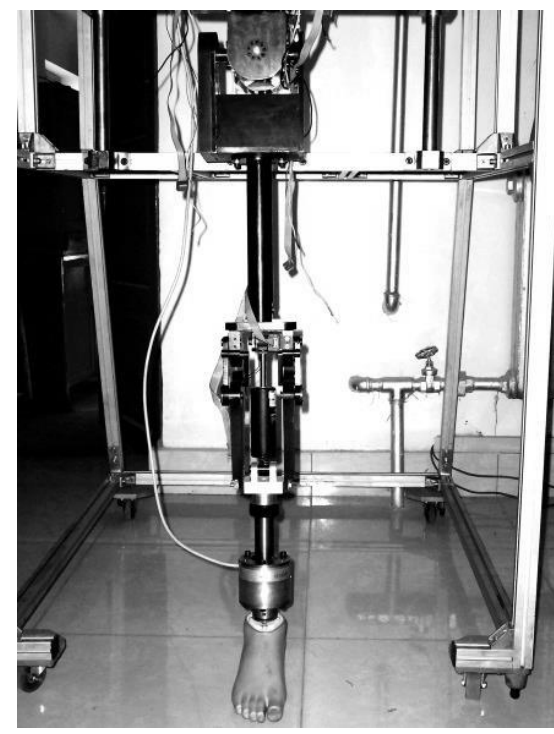

Figure 1: Prototype of the intelligent bionic leg.

It is constituted of a thigh pole, knee joint, shank pole, prosthetic foot, and the connection part between lower limb prosthesis and the human body. The knee joint is semi-actively controlled by MRD with one degree of freedom of movement. The intelligent bionic leg adopts energy-storing solid ankle prosthetic foot and its ankle joint has no degree of freedom of movement. The four-bar closed-chain multi-axis knee mechanism that is adopted by the prosthetic knee joint has the following advantages [12, 13]:

(1) The anteroposterior cruciate ligaments of human knee joints are simulated by anteroposterior bars of the knee joint, with instantaneous centre of rotation (ICR) resembling a "J" curve of a human knee joint as shown in Fig. 2. 


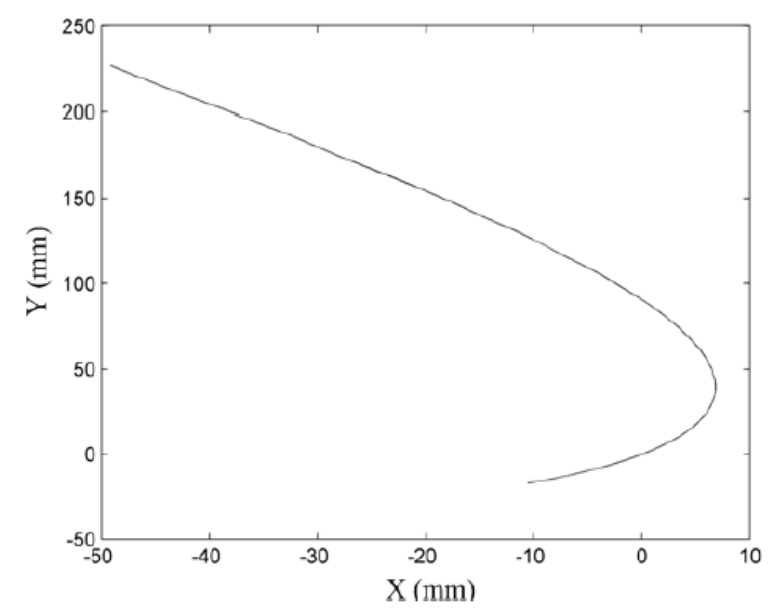

Figure 2: ICR trajectory of the four-bar closed-chain multi-axis knee mechanism.

(2) The four-bar closed-chain multi-axis knee mechanism is subject to translation and rotation. With it, the position of ankle joints is upgraded higher than that of single-axis ankle joints during the swing phase. Thus, with its larger gap to avoid collision, the intelligent bionic leg facilitates walking.

(3) As ICR is changeable, the ICR of the prosthetic knee joint first rises higher than that of a human knee joint in the support phase, and then falls rapidly in the swing phase. In the support phase, the ground reaction force (GRF) is initially at the front of ICR and the prosthesis maintains stability due to limiting blocks. GRF is at the back of ICR in the telophase, which facilitates bending in the prosthesis. Details are shown in Fig. 3.

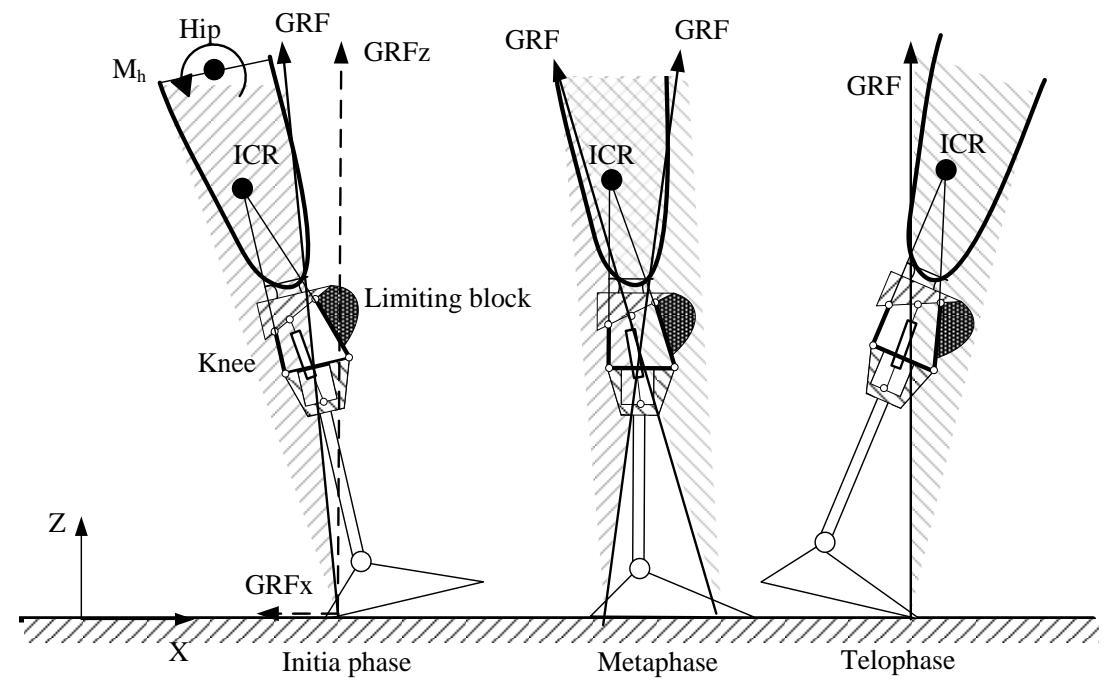

Figure 3: Support phase of intelligent bionic leg.

\section{FORWARD DYNAMICS MODELLING OF MRD}

When controlling the intelligent bionic leg based on MRD, precise MRD dynamics modelling plays an important role in choosing a control algorithm and acquiring effective control. Current dynamics models of MRD basically include two types: one is based on shear stress of magnetorheological fluid and constitutive equations of strain state, such as the Bingham equation and the Herschel-Bulkley equation [14, 15]; the other is based on the optimal algorithm in experiments. The above second model includes a non-parametric model, such as the Takagi-Sugeno model and the Chebychev polynomial model, and a parametric model, such as the hyperbolic tangent model, phenomenological model, extended nonlinear hysteretic 
bi-viscous model, general Sigmoid hysteretic model, extended hyperbolic tangent model, simplified hyperbolic tangent model, Bouc-Wen model and modified Bouc-Wen model [1619]. The Bingham model can precisely expound the relationship between the damping force of MRD and displacement, but it fails to describe the nonlinearity and hysteretic features between damping force and velocity under low velocity. The Bouc-Wen model can precisely describe the relationship between the damping force and displacement or velocity of MRD, but the model itself is complicated and it is difficult to recognize its parameters. The hyperbolic tangent model, despite its simplicity in parameter recognition and being a solution to corresponding inverse model, it lacks precision. After comprehensive consideration, the Bouc-Wen model is chosen for this research as a basis for the forward dynamics model of MRD. The Bouc-Wen model is shown in Fig. 4.

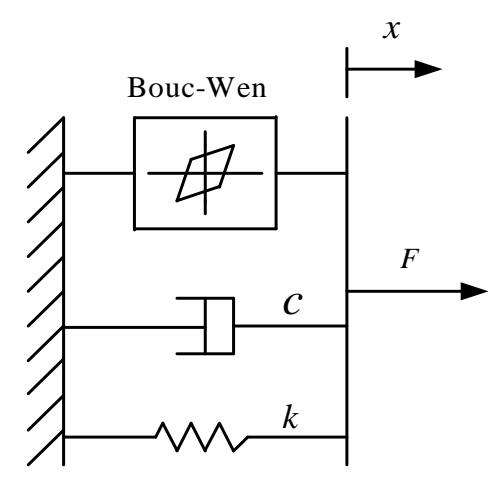

Figure 4: Bouc-Wen model of MRD.

Relative dynamics equations are:

$$
\begin{gathered}
F=c_{0} \dot{x}+k_{0}\left(x-x_{0}\right)+\alpha z \\
\dot{z}=-\gamma|\dot{x}| z|z|^{n-1}-\beta \dot{x}|z|^{n}+A \dot{x}
\end{gathered}
$$

where $F$ is the damping force of MRD, $x$ is the displacement of piston rod of MRD, $\dot{x}$ is the velocity of the piston rod of MRD, $c_{0}$ is the parameter of the damper, $k_{0}$ is the stiffness parameter of the linear spring, and $\alpha$ is the parameter decided by the control system and magnetorheological damping fluid. As coefficients, $\gamma, \beta, A, n$ are determined by the feature of magnetorheological fluid and the structure of MRD, including the nonlinear damp and stiffness of MRD.

In this paper, the expressions of the main parameters of the Bouc-Wen model are as follows [20]:

$$
\begin{gathered}
c_{0}=9 I^{2}+631 I+2183 \\
k_{0}=582 I^{2}-960 I+1048 \\
\alpha=-9718 I^{2}+653000 I+375600
\end{gathered}
$$

where $x_{0}=0, \gamma=5000000, n=2, \beta=1.75, A=3$, and $I$ represents currents.

The Simulink module in MATLAB is used to simulate the Bouc-Wen model, as shown in Fig. 5. From Eqs. (1) to (3) it can be seen that there are displacement, velocity, and currents as inputs and damping force as output in Simulink.

During simulation with Simulink under the Bonc-Wen model, a Sinusoidal signal input added to the displacement input module of the simulation system will be converted into the displacement of the piston rod toward the cylinder, and the derivative of the signal becomes the velocity of the piston rod toward the cylinder. The sinusoidal signal and its derivations are shown as follows:

$$
\begin{gathered}
x=A \sin (\omega t) \\
\dot{x}=A \omega \cos (\omega t)
\end{gathered}
$$

where $\omega=2 \pi f, A$ is the amplitude, and $f$ is the frequency. 


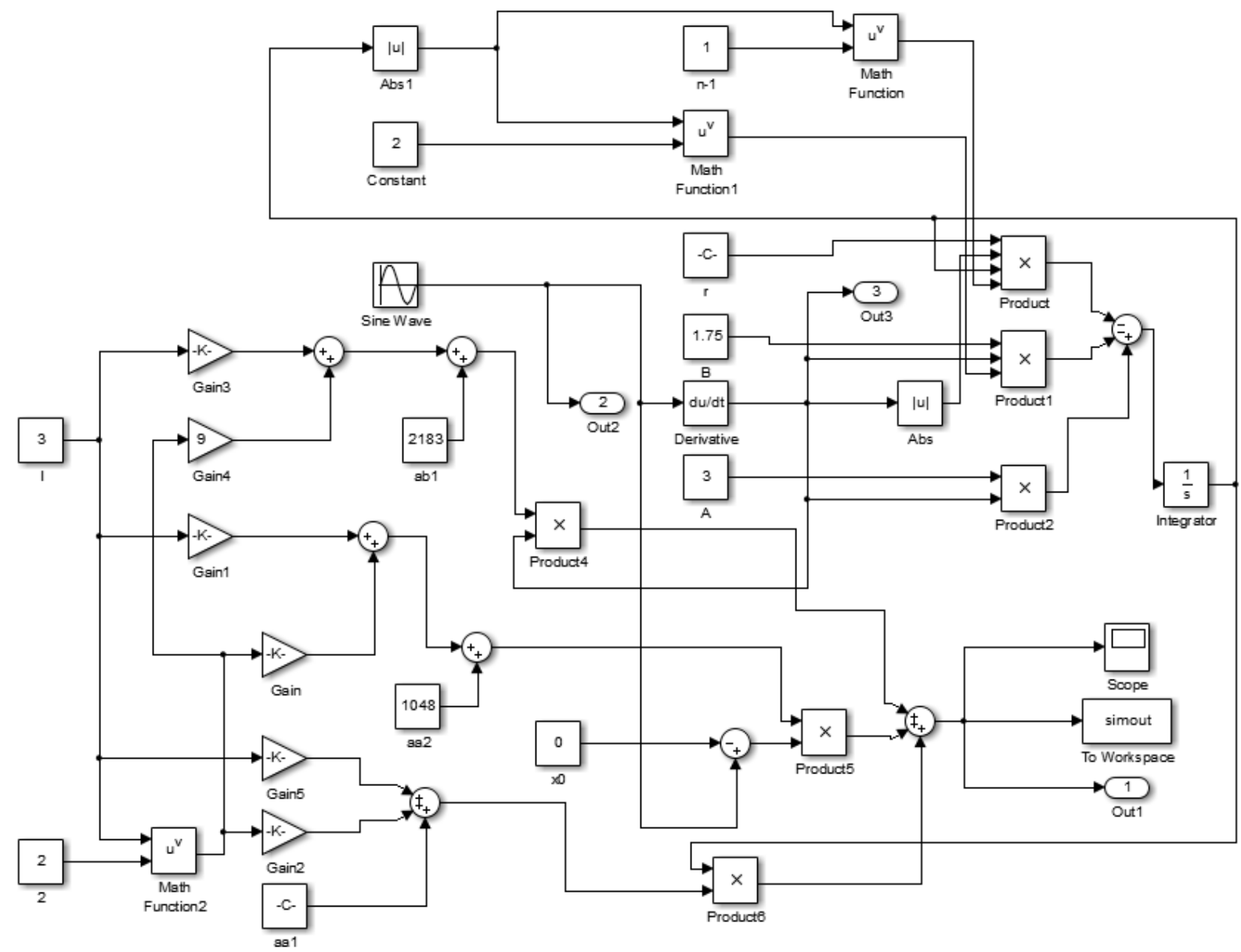

Figure 5: Simulative system of Simulink in Bouc-Wen model.

For simulation, the value of amplitude is set as $20 \mathrm{~mm}$, and the frequency is set as $1 \mathrm{~Hz}$. Values of currents are taken as $0 \mathrm{~A}, 0.4 \mathrm{~A}, 0.8 \mathrm{~A}, 1.2 \mathrm{~A}, 1.6 \mathrm{~A}$ and $2.0 \mathrm{~A}$. The simulation time is $5 \mathrm{~s}$, and the sampling frequency is $200 \mathrm{~Hz}$. The simulation results are shown in Figs. 6 to 9.

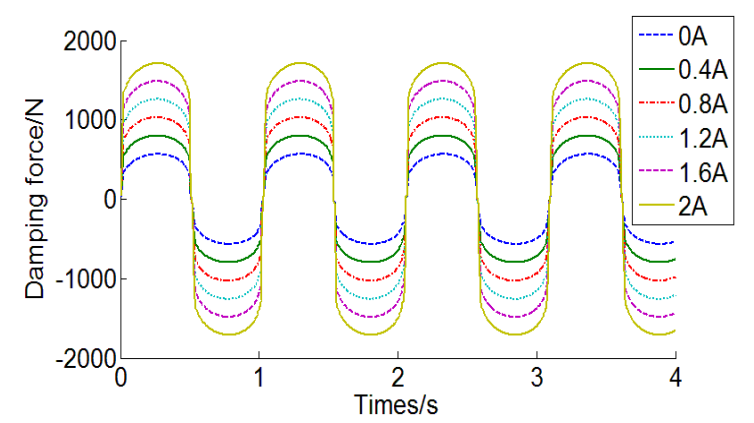

Figure 6: Curves of damping force against time.

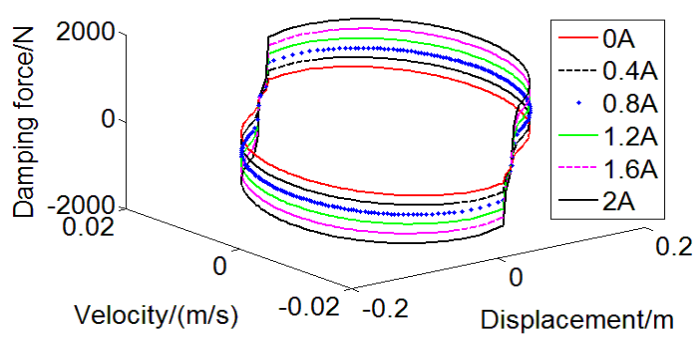

Figure 7: Curves of damping force against velocity and displacement. 


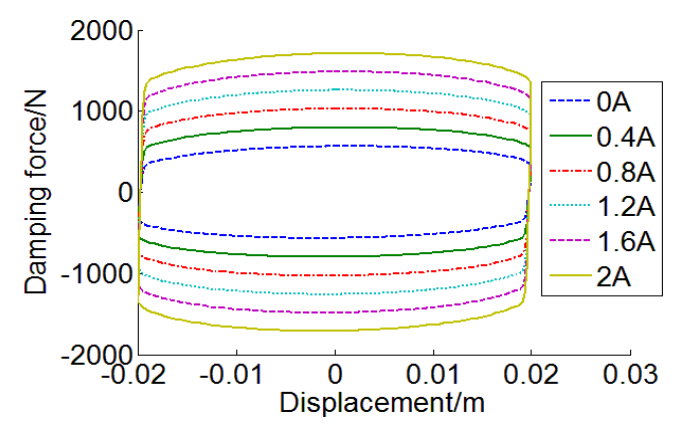

Figure 8: Curves of damping force against displacement.

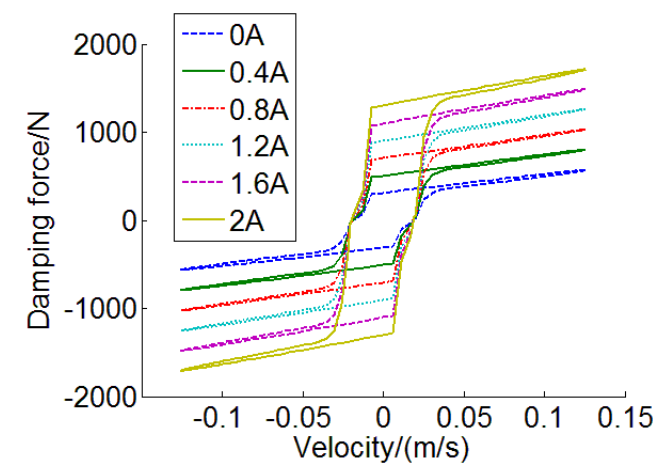

Figure 9: Curves of damping force against velocity.

Fig. 6 shows that as the current intensity increases, the output damping force of MRD gains great amplitude. Figs. 7 to 9 show that the damping force is affected both by displacement and velocity, but it is difficult to weigh their influence. Since the amplitude and frequency used in simulation are fixed, and the damping forces have relations with velocity and displacement, it is thus necessary to make separate analyses where displacement is changing toward fixed currents and vice versa.

For Simulink simulation, the value of currents is set as $2 \mathrm{~A}$, and the frequency is set as 0.5 Hz. Values of amplitudes are taken as $8 \mathrm{~mm}, 12 \mathrm{~mm}, 16 \mathrm{~mm}, 20 \mathrm{~mm}, 24 \mathrm{~mm}, 28 \mathrm{~mm}, 32 \mathrm{~mm}$, $36 \mathrm{~mm}$ and $40 \mathrm{~mm}$. The simulation time is set as $5 \mathrm{~s}$, and the sampling frequency is set as 200 Hz. Two sets of data are acquired from the simulation results: one is the varying damping force under fixed displacements and varying velocity as shown in Table I; the other is the varying damping force under a fixed velocity and varying displacement, as shown in Table II. Corresponding curves are drawn in Fig. 10 according to the above two sets of data. The straight slope in the curve between displacement and damping force is 1437.5 , and 3482.2 in the curve between velocity and damping force. Therefore, it can be deduced that the influence of velocity on damping force exceeds that of displacement.

Table I: The value of damping force under various amplitude, various velocity, and the same displacement.

\begin{tabular}{|c|c|c|c|}
\hline Amplitude $(\mathrm{m})$ & Displacement $(\mathrm{m})$ & Velocity $(\mathrm{m} / \mathrm{s})$ & Damping force $(\mathrm{N})$ \\
\hline 0.008 & 0.003985481 & 0.04366211 & 1430.242074 \\
0.012 & 0.003993835 & 0.071178178 & 1526.037677 \\
0.016 & 0.003979038 & 0.097450502 & 1617.470091 \\
0.02 & 0.0039942 & 0.123210249 & 1707.161847 \\
0.024 & 0.004052003 & 0.148710708 & 1796.013107 \\
0.028 & 0.004032302 & 0.174173772 & 1884.621348 \\
0.032 & 0.004010663 & 0.199554351 & 1972.939637 \\
0.036 & 0.00406283 & 0.224828302 & 2060.994213 \\
0.04 & 0.004014469 & 0.250136061 & 2149.02011 \\
\hline
\end{tabular}


Table II: The value of damping force under various amplitude, various displacement, and the same velocity.

\begin{tabular}{|c|c|c|c|}
\hline Amplitude $(\mathrm{m})$ & Velocity $(\mathrm{m} / \mathrm{s})$ & Displacement $(\mathrm{m})$ & Damping force $(\mathrm{N})$ \\
\hline 0.008 & 0.024904428 & 0.00696147 & 1369.279629 \\
0.012 & 0.024870471 & 0.011340757 & 1375.537668 \\
0.016 & 0.025306774 & 0.015497331 & 1383.108408 \\
0.02 & 0.024709301 & 0.01962181 & 1387.033848 \\
0.024 & 0.024992377 & 0.023680463 & 1393.928634 \\
0.028 & 0.024788599 & 0.02773292 & 1399.119658 \\
0.032 & 0.024572904 & 0.031772243 & 1404.25008 \\
0.036 & 0.024821276 & 0.035794828 & 1410.971545 \\
0.04 & 0.024437905 & 0.039822479 & 1415.50129 \\
\hline
\end{tabular}

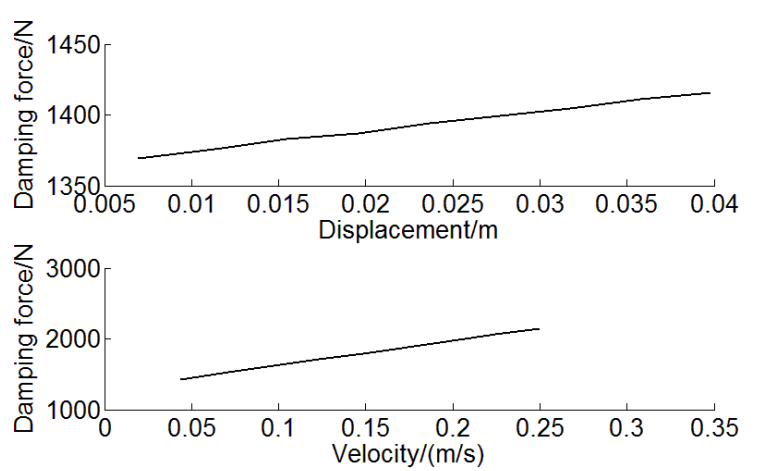

Figure 10: The corresponding curve between damping force and displacement $\&$ velocity.

\section{INVERSE DYNAMICS MODELLING OF MRD}

When the working condition and value of the input current of MRD are informed, the output damping force can then be acquired under the established forward dynamics model. However, if MRD is used for control, the value of input currents has to be determined according to the working condition and desired damping force. So an inverse dynamics model of MRD is required.

Although scholars have proposed numerous forward dynamics models of MRD, the common feature of strong non-linearity in most of these models raises the difficulty of calculating the inverse model, which can hardly be accomplished through analytic methods. Even with the small amount of inverse model of the forward dynamics that can be obtained through analytic methods, still these models are usually simple and lack precision in solution. In contrast, a neural network has strong nonlinear processing capacity, and can map the relationships of complex nonlinear functions. Therefore, the BP neural network is chosen in this work to establish an inverse dynamics model of MRD.

\subsection{Data collection in training and testing BP neural network}

Since numerous data is required in training and testing the BP neural network, simulation is thus undertaken according to the established Bouc-Wen model. To guarantee that the inverse dynamics model can precisely identify all currents that suit all of the working conditions, the following methods are needed to obtain numerous and comprehensive training and testing data.

A highly efficient method to obtain numerous data samples is using a random sequence as an input signal. Gaussian white noise under a low pass filtering (a random sequence that complies with normal distribution) can be adopted as the input data for training. Since velocity is the derivative of displacement, both can be regarded as the same parameter. The 
displacement, velocity, and current signal for training are shown in Table III. The displacement, velocity, and current signal for testing are shown in Table IV.

Table III: Training data for BP neural network.

\begin{tabular}{|l|c|c|c|c|}
\hline Signal $\backslash$ Time length $(\mathrm{s})$ & $0 \sim 10$ & $10 \sim 20$ & $20 \sim 30$ & $30 \sim 40$ \\
\hline Displacement $(\mathrm{m})$ & GWN1 & GWN1 & GWN1 & GWN1 \\
Velocity (m/s) & GWN2 & GWN2 & GWN2 & GWN2 \\
Current (A) & GWN3 & $\sin (4 \pi t)+1$ & 0 & 2 \\
\hline
\end{tabular}

GWN1: Gaussian white noise under low pass filtering, frequency: 0 6 Hz, amplitude: $\pm 0.04 \mathrm{~m}$. GWN2: Derivative of GWN1 toward time, frequency: $0 \sim 6 \mathrm{~Hz}$, amplitude: $\pm 0.5 \mathrm{~m} / \mathrm{s}$.

GWN3: Gaussian white noise under low pass filtering, frequency: 0 15 Hz, amplitude: 0 2 A.

Table IV: Testing data for BP neural network.

\begin{tabular}{|l|c|c|c|}
\hline Signal $\backslash$ Time length $(\mathrm{s})$ & $0 \sim 5$ & $5 \sim 10$ & $10 \sim 15$ \\
\hline Displacement $(\mathrm{m})$ & GWN1 & GWN1 & $0.03 \times \sin (2 \pi \mathrm{t})$ \\
Velocity $(\mathrm{m} / \mathrm{s})$ & GWN2 & GWN2 & $0.03 \times \sin (2 \pi \mathrm{t})$ \\
Current $(\mathrm{A})$ & GWN3 & $\sin (2 \pi \mathrm{t})+1$ & $\mathrm{GWN3}$ \\
\hline
\end{tabular}

GWN1: Gaussian white noise under low pass filtering, frequency: 0 3 Hz, amplitude: $\pm 0.02 \mathrm{~m}$.

GWN2: Derivative of GWN1 toward time, frequency: $0 \sim 3 \mathrm{~Hz}$, amplitude: $\pm 0.1 \mathrm{~m} / \mathrm{s}$.

GWN3: Gaussian white noise under low pass filtering, frequency: 0 6 Hz, amplitude: 0 2 A.

\subsection{Construction and testing of BP neural network}

In order to construct the BP neural network, the number of input vector and output vector, levels of BP neural network, the number of latent neuron, initial weight, threshold, and transfer functions must all first be determined. The BP neural network designed herein includes input level, output level, and double latent levels. The output damping force of MRD is the function of the piston displacement, the piston velocity, and the electric currents, so the following variables are chosen for this work as 7 nodes of the input level: damping force, displacement, and velocity at the present time, and damping force, displacement, velocity, and currents at previous time. There is only the current at the present time as the node of the output level. Eq. (6) determines the number of nodes in one single latent level.

$$
l<\sqrt{(m+n)+a}
$$

where $l$ is the number of nodes in the latent level, $m$ is the number of nodes in the output level, $n$ is the number of nodes in the input level, $a$ is the coefficient with its value ranging from 0 to 10 . Considering the strong nonlinearity of the Bouc-Wen model, the nodes of the double latent levels are taken respectively as 10 and 5 for better training results.

Logsig and Purelin are respectively the input and output function of the BP neural network. The initial weight and threshold use the default values. The learning velocity and training expectations are set as needed. Based on the above condition, the training function of the BP neural network model is as follows:

$$
\text { net=newff(inputn,outputn,[10 5],\{ 'logsig' 'purelin' \}) }
$$

The training data in the BP neural network is taken from signals in Table III, where the sampling frequency is $1,000 \mathrm{~Hz}$, and the number of sampling is 40,000 . Testing data is from signals in Table IV, where the sampling frequency is $1,000 \mathrm{~Hz}$. This work uses testing data to test the trained BP neural network, whose results are shown in Fig. 11. It can be seen that the maximum testing error of $0.0168 \mathrm{~A}$, satisfies the requirement for the desired accuracy and that the actual current can follow the desired current well. 

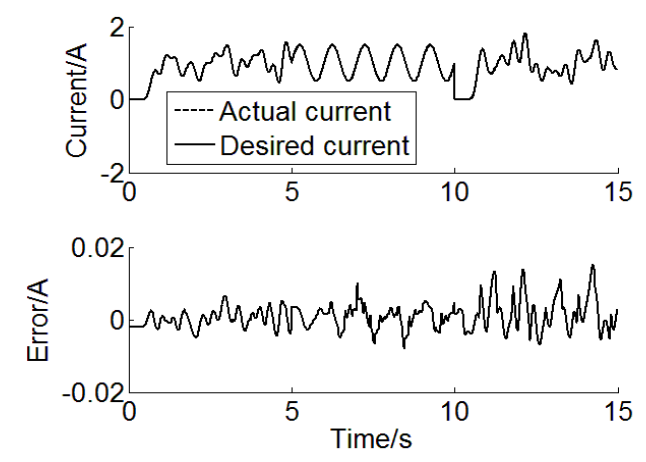

Figure 11: Testing results of custom signals.

\section{SIMULATION OF KNEE JOINT MOVEMENT CONTROL}

Based on SolidWorks, ADAMS, and MATLAB/Simulink, this paper establishes the simulation platform for integrated control on knee joints of intelligent bionic leg. The threedimensional model of the intelligent bionic leg in SolidWorks is shown in Fig. 12. Data of the model is then transferred into ADAMS to add various constraints and to determine its material properties, and is finally transferred into MATLAB/Simulink for simulation.

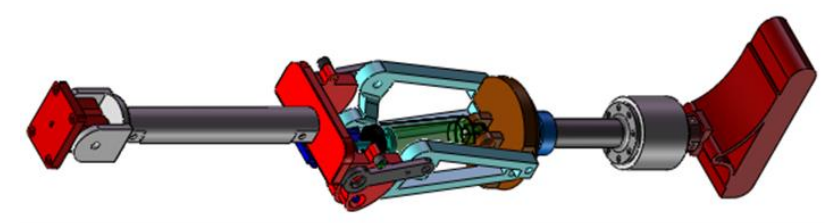

Figure 12: The three-dimensional model of intelligent bionic leg.

\subsection{Design of RBF neural network controller}

The dynamics equation of the intelligent bionic leg with $\mathrm{n}$ joints is as follows:

$$
M(q) \ddot{q}+V(q, \dot{q}) \dot{q}+G(q)+\Delta(q, \dot{q})+d=T
$$

where $M(q)$ is the positive definite inertia matrices of $n \times n$ order, $V(q, \dot{q})$ is the centrifugal force and Coriolis force item of $n \times n$ order, $G(q)$ is the gravity item of $n \times 1$ order, $q$ represents the vector of joint variable, $T$ is the joint torque vector exerted on actuator, $\Delta(q, \dot{q})$ is the uncertainty of the model, and $d$ is outer disturbance.

Supposing the ideal tracking trajectory is $q_{d}$, and the tracking error is defined as $e=q-q_{d}$, then the designed feed forward control law is as follows:

$$
T=u+M(q) \ddot{q_{d}}+V(q, \dot{q}) \dot{q}_{d}+G(q)
$$

where $u$ is the feedback control law.

By inserting Eq. (8) into Eq. (7), it can be derived that

$$
M(q) \ddot{e}+V(q, \dot{q}) \dot{e}+\Delta(q, \dot{q})+d=u
$$

Make $\Delta f(q, \dot{q})=\Delta(q, \dot{q})+d$, then

$$
M(q) \ddot{e}+V(q, \dot{q}) \dot{e}+\Delta f=u
$$

$\Delta f$ is approached using RBF neural network as follows:

$$
\Delta f=W_{f}^{*} \sigma_{f}+\varepsilon_{f}
$$

where $\varepsilon_{f}$ is the approximation error, $\sigma_{f}$ is the RBF Gaussian function, and $W_{f}^{*}$ is the weight of the ideal neural network. 
By combining eq. (10) with eq. (11) it is acquired that

$$
M(q) \ddot{e}+V(q, \dot{q}) \dot{e}+W_{f}^{*} \sigma_{f}+\varepsilon_{f}=u
$$

By the following definition

$$
\left\{\begin{array}{c}
x_{1}=e \\
x_{2}=\dot{e}+\alpha e
\end{array}\right.
$$

where $\alpha>0$.

It can be derived that

$$
\left\{\begin{array}{c}
\dot{x}_{1}=x_{2}-\alpha x_{1} \\
M \dot{x}_{2}=-V x_{2}+\omega-W_{f}^{*} \sigma_{f}-\varepsilon_{f}+u
\end{array}\right.
$$

where $\omega=M \alpha \dot{e}+V \alpha e$.

For Eq. (14), the self-adaptive law is designed as

$$
\dot{W}_{f}=-\eta x_{2} \sigma_{f}^{T}
$$

And the feedback control law is designed as

$$
u=-\omega-\frac{1}{2 \gamma^{2}} x_{2}+W_{f} \sigma_{f}-\frac{1}{2} x_{2}
$$

where $W_{f}$ and $\sigma_{f}$ are respectively the weight and Gaussian function output of the neural network.

According to Eqs. (8), (15) and (16), the structure of RBF neural network in the knee joint controller designed herein is 2-5-1 RBF, where the control parameters are $\eta=1000, \alpha=10$ and $\gamma=0.05$. The parameters of the Gaussian function are $c_{i}=\left[\begin{array}{lllll}-1 & -0.5 & 0 & 0.5 & 1\end{array}\right]$ and $b_{i}=50$. The RBF neural network controller is implemented by MATLAB programming, whose function module is shown in Fig. 13.

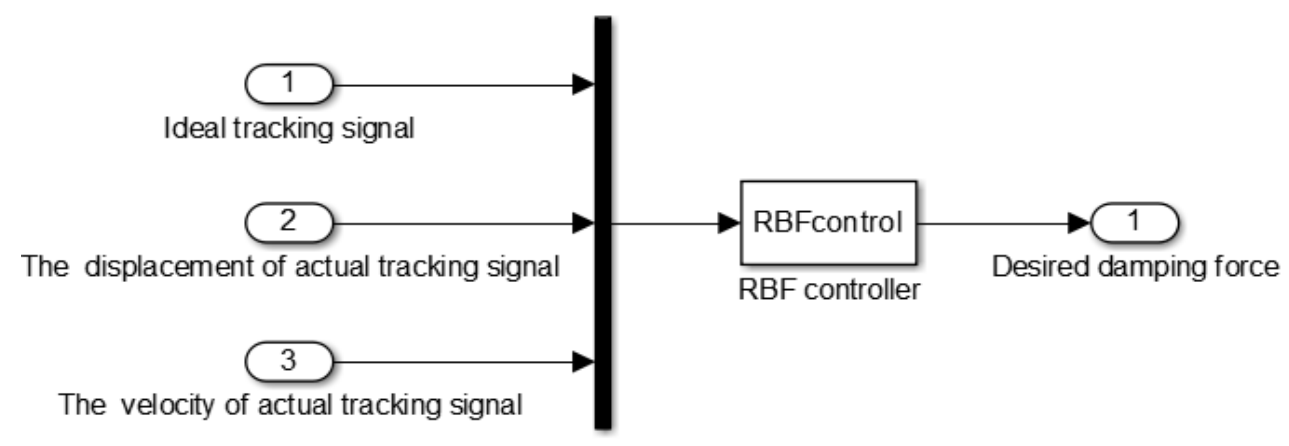

Figure 13: RBF neural network controller.

\subsection{Simulation and analysis on integrated control}

The established RBF neural network control system of the intelligent bionic leg in MATLAB/Simulink based on the forward and inverse dynamics models of MRD is shown in Fig. 14. In Fig. 14, adams-sub represents a packetized document of the intelligent bionic leg in ADAMS.

The given equation of the ideal curve of knee joint movement is as follows:

$$
y=\sin (t)+1.2
$$

The simulation time ranges from $4.8 \mathrm{~s}$ to $20 \mathrm{~s}$. The tracking curve and error curve of the knee joint movements are acquired as shown in Figs. 15 and 16. The curves of ideal damping force and actual damping force are shown in Fig. 17. And the curve of control currents is shown in Fig. 18. 


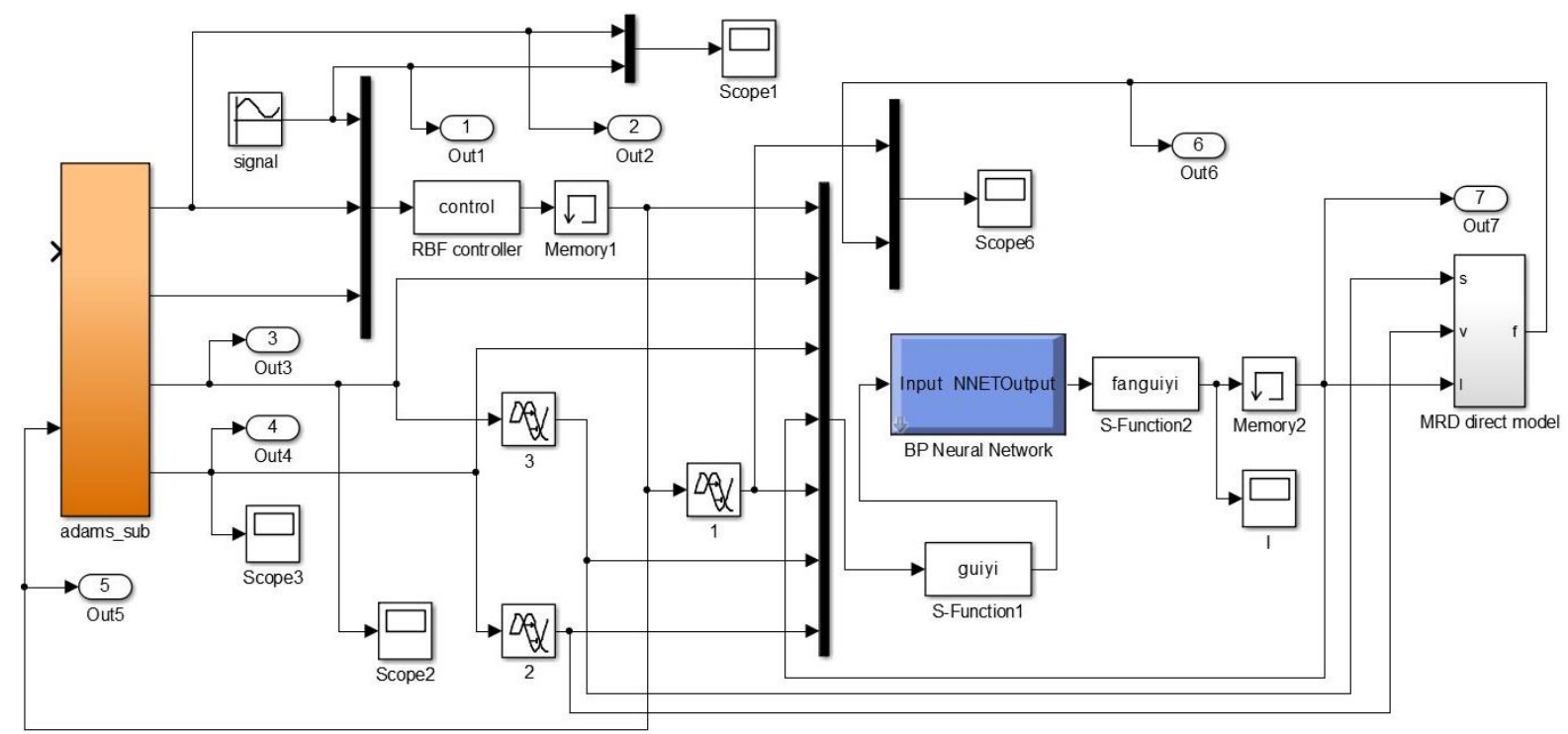

Figure 14: The simulation platform for knee joint integrate control of intelligent bionic leg.

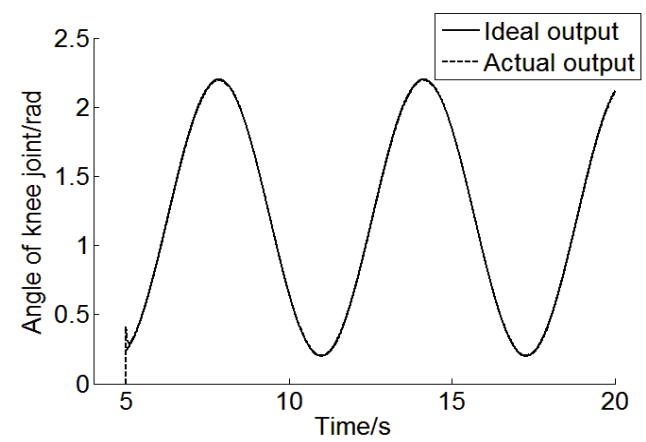

Figure 15: The tracking curve of knee joint movement.
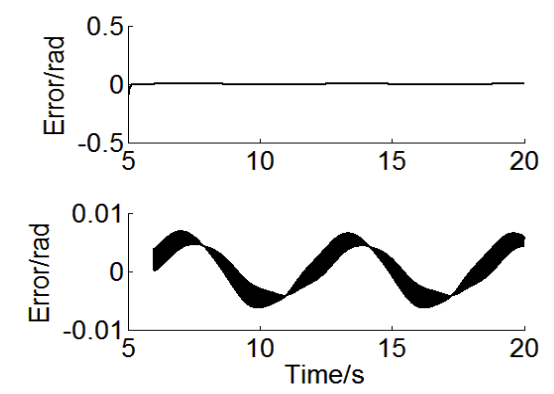

Figure 16: The tracking error curve of knee joint movement.

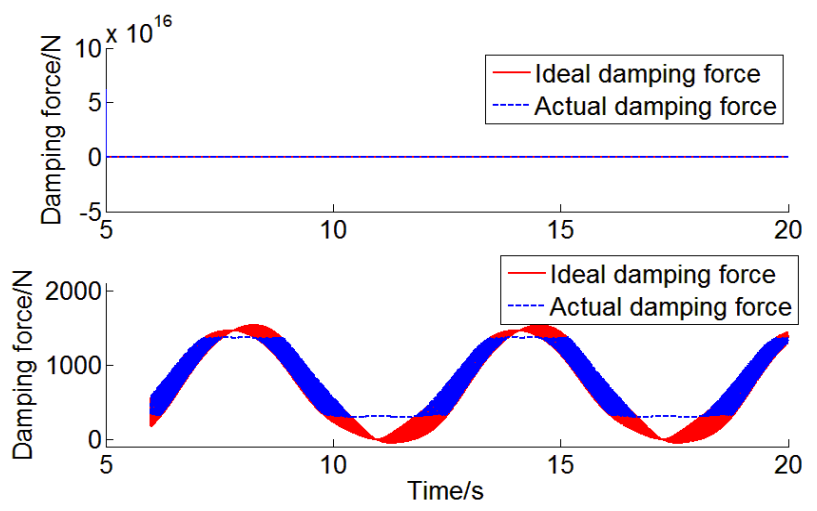

Figure 17: The ideal and actual damping force curve. 


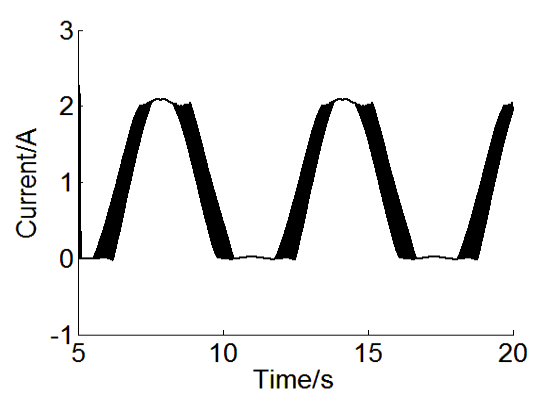

Figure 18: Control currents curve.

From Figs. 15 and 16, it is seen that under the control of the RBM neural network controller, the knee joint movement can well track the ideal movement curve. Since there is certain angular deviation between the initial positions of knee joint movement and the follow curve, the curve of knee joint movement shows major fluctuation at the initial stage of the movement.

As shown in Fig. 17, there is large fluctuation of the ideal damping force at the initial control stage, but it tends to be smooth at the rest of the control stage. Restricted by the structure and materials of MRD, there are upper and lower limits for the damping force that MRD provides. Therefore, when the actual damping force is beyond this scope, the damper can only output its own highest and lowest damping force. Fig. 18 shows that most of the currents range within the scope of rated currents (0-2 A).

\section{CONCLUSION}

By using a four-bar closed-chain multi-axis knee joint, the designed intelligent bionic leg acquires good traits. The established forward dynamics model of Bouc-Wen in MRD can not only relatively precisely describe the relationship between damping force and velocity as well as between damping force and displacement, but also proves that the influence of velocity on damping force is greater than that of displacement. Based on simulative data of the forward dynamics model of MRD, the inverse model of BP neural network can well anticipate the value of input currents of MRD. The combined controlling simulation shows that the intelligent bionic leg can better track the desired gait, and that the RBF neural network control algorithm achieves good control effects. The forward and inverse dynamics model of MRD can better describe the features of MRD.

\section{ACKNOWLEDGEMENT}

This work was supported by the National Natural Science Foundation of China (Grant No.51105070, 51505072), Basic Research Project of Key Laboratory of Education Department of Liaoning Province (Grant No. LZ2015037) and the Fundamental Research Funds for the Central Universities of the Ministry of Education of China (Grant No. N140305001). We are sincerely grateful for the support.

\section{REFERENCES}

[1] Gong, S.-Y.; Yang, P.; Song, L.; Chen, L.-L. (2011). Simulation of swing phase dynamics in trans-femoral prostheses based on MATLAB, Journal of Hebei University of Technology, Vol. 40, No. 2, 6-9, doi:10.3969/j.issn.1007-2373.2011.02.002

[2] Pillai, M. V.; Kazerooni, H.; Hurwich, A. (2011). Design of a semi-active knee-ankle prosthesis, Proceedings of the IEEE International Conference on Robotics and Automation, 5293-5300, doi:10.1109/ICRA.2011.5980178 
[3] Ziegler-Graham, K.; MacKenzie, E. J.; Ephraim, P. L.; Travison, T. G.; Brookmeyer, R. (2008). Estimating the prevalence of limb loss in the United States: 2005 to 2050, Archives of Physical Medicine and Rehabilitation, Vol. 89, No. 3, 422-429, doi:10.1016/j.apmr.2007.11.005

[4] Pejhan, S.; Farahmand, F.; Parnianpour, M. (2008). Design optimization of an above-knee prosthesis based on the kinematics of gait, Proceedings of the $30^{\text {th }}$ Annual International Conference of the IEEE Engineering in Medicine and Biology Society, 4274-4277, doi:10.1109/IEMBS.2008.4650154

[5] Waycaster, G.; Wu, S.-K.; Shen, X. (2011). Design and control of a pneumatic artificial muscle actuated above-knee prosthesis, Journal of Medical Devices, Vol. 5, No. 3, Paper 031003, 9 pages, doi: $10.1115 / 1.4004417$

[6] Sodeyama, H.; Sunakoda, K.; Fujitani, H.; Soda, S.; Iwata, N.; Hata, K. (2003). Dynamic tests and simulation of magneto-rheological dampers, Computer-Aided Civil and Infrastructure Engineering, Vol. 18, No. 1, 45-57, doi:10.1111/1467-8667.t01-1-00298

[7] Akdoğan, K. E.; Yılmaz, A.; Sadeghimorad, A.; Şahin, İ. (2012). Design of semi active knee joint with magnetorheological (MR) damper, Proceedings of the $20^{\text {th }}$ Signal Processing and Communications Applications Conference, 1-4, doi:10.1109/SIU.2012.6204680

[8] Kim, J.-H.; Oh, J.-H. (2001). Development of an above knee prosthesis using MR damper and leg simulator, Proceedings of the IEEE International Conference on Robotics \& Automation, Vol. 4, 3686-3691, doi:10.1109/ROBOT.2001.933191

[9] Herr, H.; Wilkenfeld, A. (2003). User-adaptive control of a magnetorheological prosthetic knee, Industrial Robot: An International Journal, Vol. 30, No. 1, 42-55, doi:10.1108/ 01439910310457706

[10] Wang, H.; Simpson, K. J.; Chamnongkich, S.; Kinsey, T.; Mahoney, O. M. (2005). A biomechanical comparison between the single-axis and multi-axis total knee arthroplasty systems for the stand-to-sit movement, Clinical Biomechanics, Vol. 20, No. 4, 428-433, doi: 10.1016/j.clinbiomech.2004.12.003

[11] Xie, H.-L.; He, N.; Li, F.; Yang, J.-Y. (2015). The bionic design and system identification of intelligent bionic leg with magneto-rheological damper, Technical Gazette, Vol. 22, No. 5, 1093 1098, doi:10.17559/TV-20150731100315

[12] Wang, B.-R.; Jin, Y.-L.; Xu, H.; Xu, X.-H. (2008). Computed torque and proportion-differential feedback control for robot bionic knee joint, Chinese Journal of Mechanical Engineering, Vol. 44, No. 1, 179-183, doi:10.3321/j.issn:0577-6686.2008.01.031

[13] Zahedi, S.; Sykes, A.; Lang, S.; Cullington, I. (2005). Adaptive prosthesis - a new concept in prosthetic knee control, Robotica, Vol. 23, No. 3, 337-344, doi:10.1017/S0263574704001365

[14] Hong, S. R.; Wereley, N. M.; Choi, Y. T.; Choi, S. B. (2008). Analytical and experimental validation of a nondimensional Bingham model for mixed-mode magnetorheological dampers, Journal of Sound and Vibration, Vol. 312, No. 3, 399-417, doi:10.1016/j.jsv.2007.07.087

[15] Wereley, N. M. (2008). Nondimensional Herschel-Bulkley analysis of magnetorheological and electrorheological dampers, Journal of Intelligent Material Systems and Structures, Vol. 19, No. 3, 257-268, doi:10.1177/1045389X07088107

[16] Li, H. N.; Yang, H.; Li, X. L. (2004). Advances of research on parameterized dynamic models of magnetorheological dampers, Journal of Dalian University of Technology, Vol. 44, No. 4, 616624, doi:10.3321/j.issn:1000-8608.2004.04.033

[17] Kwok, N. M.; Ha, Q. P.; Nguyen, M. T.; Li, J.; Samali, B. (2007). Bouc-Wen model parameter identification for a MR fluid damper using computationally efficient GA, ISA Transactions, Vol. 46, No. 2, 167-179, doi:10.1016/j.isatra.2006.08.005

[18] Spencer Jr., B. F.; Dyke, S. J.; Sain, M. K.; Carlson, J. D. (1997). Phenomenological model for magnetorheological dampers, Journal of Engineering Mechanics, Vol. 123, No. 3, 230-238, doi:10.1061/(ASCE)0733-9399(1997)123:3(230)

[19] Lange, U.; Richter, L.; Zipser, L. (2001). Flow of magnetorheological fluids, Journal of Intelligent Material Systems and Structures, Vol. 12, No. 3, 161-164, doi:10.1106/PF05-DTU22QTD-28B6

[20] Liu, Y.-Q.; Yang, S.-P.; Liao, Y.-Y.; Zhang, G.-N. (2011). Parameter identification of Bouc-Wen model for MR damper based on genetic algorithm, Journal of Vibration and Shock, Vol. 30, No. 7, 261-265, doi:10.3969/j.issn.1000-3835.2011.07.051 